\title{
Full Term Birth
}

National Cancer Institute

\section{Source}

National Cancer Institute. Full Term Birth. NCI Thesaurus. Code C114095.

Birth at 39 weeks and 0 days through 40 weeks and 6 days. 